\title{
QPSK DS-CDMA System over Rayleigh Channel with a Randomly-Varying Frequency Narrow-Band Interference: Frequency Tracking Analysis
}

\author{
Aloys N. Mvuma \\ School of Informatics, University of Dodoma, Dodoma, Tanzania \\ E-mail: \{anmvuma,mvuma\}@udom.ac.tz \\ Received August 25, 2009; revised September 7, 2009; accepted September 21, 2009
}

\begin{abstract}
This paper analyses frequency tracking characteristics of a complex-coefficient adaptive infinite-impulse response (IIR) notch filter used for suppression of narrow-band interference (NBI) with a randomly-varying frequency in a quadriphase shift keying (QPSK) modulated direct-sequence code-division multiple-access (DS-CDMA) communication system. The QPSK DS-CDMA signals are transmitted over a frequency non-selective Rayleigh fading channel. The analysis is based on a first-order real-coefficient difference equation with respect to steady-state instantaneous frequency tracking error from which a closed-form expression that relates frequency tracking mean square error (MSE) with number of DS-CDMA active users and NBI power is obtained. Closed-form expressions for optimum notch bandwidth coefficient and step size constant that minimize the frequency tracking MSE are also derived. Computer simulations are included to substantiate the accuracy of the analyses.
\end{abstract}

Keywords: Code-Division Multiple Access (CDMA), Quadriphase Shift Keying (QPSK), Complex Adaptive IIR Notch Filter, Narrow-Band Interference (NBI), Frequency Tracking MSE

\section{Introduction}

Direct-sequence code-division multiple-access (DS-CDMA) is a preferred multiplexing technique in cellular telecommunications services as it exhibits desired features that are not inherently found in other multiple access techniques, i.e., time-division multiple-access (TDMA) and frequency-division multiple-access (FDMA). Some of superior DS-CDMA features include robustness in multipath fading environment, flexibility in allocation of channels and increased spectral efficiency due to its capability of sharing bandwidth with narrow-band communication systems [1-3]. The bandwidth sharing capability is made possible by the inherent narrow-band interference (NBI) suppression capacity of DS-CDMA due to the processing gain of spread spectrum systems. However, for high levels of NBI power, the NBI suppression capacity of DS-CDMA system can be enhanced by means of signal processing techniques at the receiver. Several methods with varying complexities have been proposed for suppression of NBI in DS-CDMA communication systems [4-9].
In [10], a complex coefficient adaptive notch filter implemented as a constrained infinite-impulse response (IIR) filter with a complex Gauss-Newton adaptation algorithm was proposed. Its application in the suppression of NBI in quadriphase shift keying (QPSK) direct-sequence spread-spectrum (DSSS) communication system was shown to result in a better signal-to-noise ratio (SNR) improvement factor than that achieved by finite-impulse response (FIR) adaptive prediction filter. In [11] a complex coefficient adaptive IIR notch filter with a simplified gradient-based algorithm that does not require any matrix inversion was presented. Analyses of its convergence, steady-state and tracking characteristics were presented in [12-15], respectively. Its application in suppression of NBI in QPSK-DSSS system with fixed unknown frequency was introduced in [13].

This paper investigates frequency tracking characteristics of the complex-coefficient adaptive IIR notch filter in [11] that is used for suppression of NBI with randomly-varying frequency in a synchronous QPSK DSCDMA communication system communicating over a frequency non-selective Rayleigh fading channel. The analysis is based on the derived first-order real-coeff- 
icient difference equation with respect to steady-state instantaneous frequency tracking error from which a closed-form expression for frequency tracking mean square error (MSE) is obtained. In addition, closed-form expressions for optimum notch bandwidth coefficient and step size constant are also derived. Computer simulations are included to substantiate accuracy of the analyses.

This paper is organized as follows. Section 2 presents the system model. Complex coefficient adaptive IIR notch filter is presented in Section 3 whereas frequency tracking is analyzed in Section 4. In Section 5 simulations are presented and discussed before a conclusion in Section 6 .

\section{System Model}

We consider a synchronous DS-CDMA system over frequency-nonselective Rayleigh fading channel using QPSK modulation, quaternary pseudo-noise (PN) spreading $\mathrm{m}$-sequences and the rectangular chip waveform. It is assumed that there are $\mathrm{K}$ simultaneously transmitting users. Referring to Figure 1, the transmitted signal for the $i$-th user $\left.s_{i}(t)\right|_{i=1} ^{K}$ is expressed as

$$
\begin{aligned}
s_{i}(t)= & \sqrt{P} b_{i}^{I}(t) c_{i}^{I}(t) \cos \left(\omega_{c} t+\theta\right) \\
& +\sqrt{P} b_{i}^{Q}(t) c_{i}^{Q}(t) \sin \left(\omega_{c} t+\theta\right)
\end{aligned}
$$

where:

$P$ is the power of the transmitted signal.

$b_{i}^{I}(t)$ and $b_{i}^{Q}(t)$ are in-phase and quadrature-phase binary data signals, respectively.

$c_{i}^{I}(t)$ and $c_{i}^{Q}(t)$ are in-phase and quadrature-phase PN spectrum spreading signals, respectively.

$\omega_{c}$ and $\theta$ are the modulator carrier frequency and phase, respectively.

We define the rectangular pulse $p_{T}(t)$ with duration $T$ as

$$
p_{T}(t)= \begin{cases}1, & 0 \leq t<T \\ 0, & \text { elsewhere }\end{cases}
$$

Therefore the in-phase and quadrature-phase data and spectrum spreading signals are expressed as

$$
b_{i}^{I}(t)=\sum_{j=-\infty}^{\infty} b_{i, j}^{I} p_{T_{s}}\left(t-j T_{s}\right),
$$
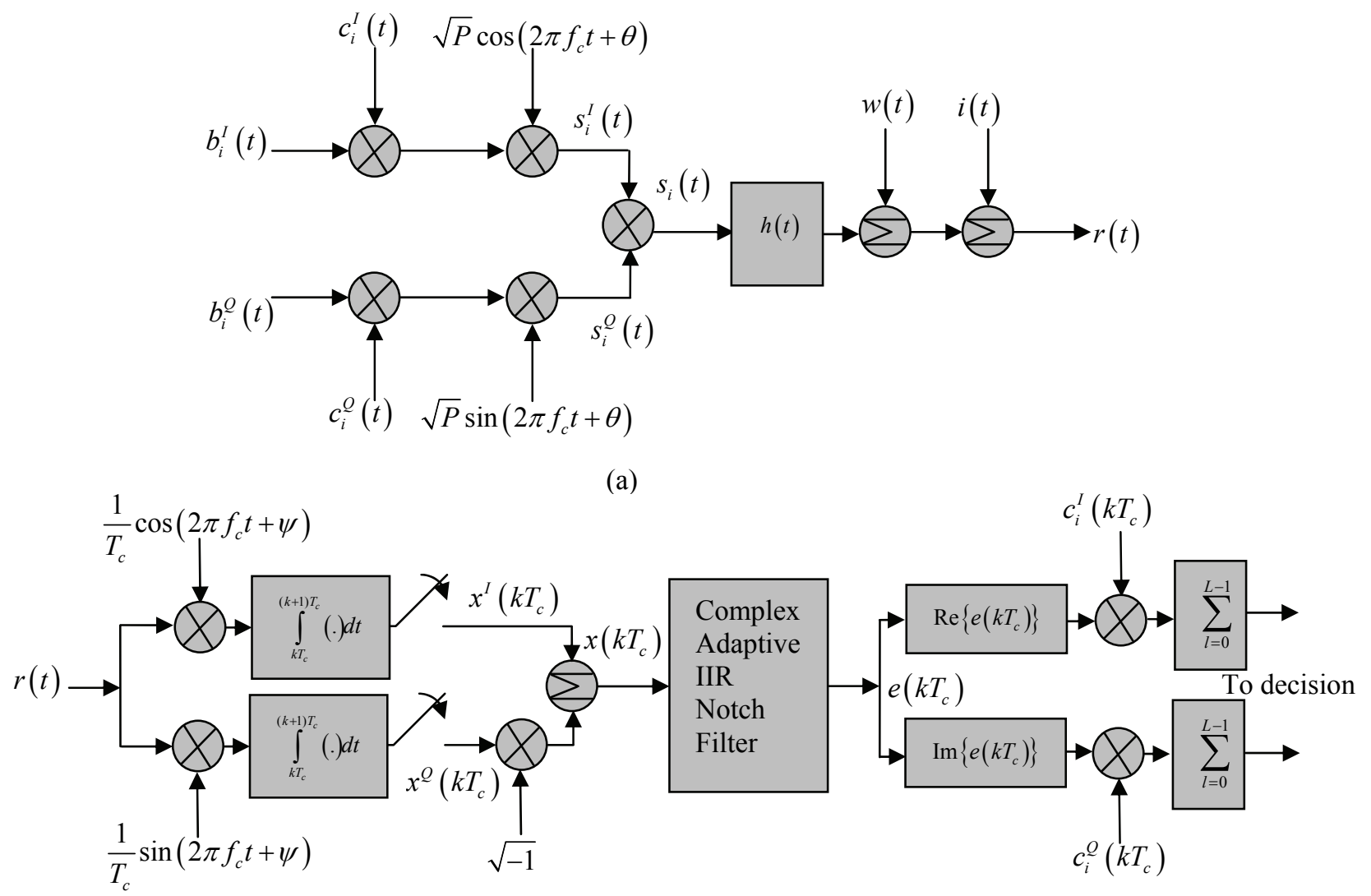

(b)

Figure 1. QPSK DS-CDMA communications system model. (a) Transmitter; (b) Receiver. 


$$
\begin{aligned}
& b_{i}^{Q}(t)=\sum_{j=-\infty}^{\infty} b_{i, j}^{Q} p_{T_{s}}\left(t-j T_{s}\right) \\
& c_{i}^{I}(t)=\sum_{j=-\infty}^{\infty} c_{i, j}^{I} p_{T_{c}}\left(t-j T_{c}\right), \\
& c_{i}^{Q}(t)=\sum_{j=-\infty}^{\infty} c_{i, j}^{Q} p_{T_{c}}\left(t-j T_{c}\right)
\end{aligned}
$$

where

$b_{i, j}^{I} \in\{+1,-1\}$ and $b_{i, j}^{Q} \in\{+1,-1\}$ are identically and independently distributed (IID) random $j$-th data bits of the $i$-th user for the in-phase and quadrature-phase components.

$c_{i, j}^{I} \in\{+1,-1\}$ and $c_{i, j}^{Q} \in\{+1,-1\}$ are IID random $j$-th chips for the $i$-th user for the in-phase and quadrature-phase components, respectively.

$T_{c}$ and $T_{s}$ are the chip duration and the symbol duration, respectively, where $T_{s} / T_{c}=L$ is the number of chips per symbol or processing gain.

The QPSK DS-CDMA signal comprising of signals for all $K$ active users is transmitted over a frequency non-selective Rayleigh fading channel with impulse response given by

$$
h(t)=\alpha \exp (j \vartheta) \delta(t-\tau)
$$

where $\vartheta$ is the phase shift with uniform PDF over $[0,2 \pi), \tau$ is the time delay which is uniformly distributed over $\left[0, T_{s}\right)$ and $\alpha$ is the Rayleigh distributed attenuation having a probability density function (PDF) expressed as

$$
f_{A}(\alpha)= \begin{cases}\alpha \exp \left(-\frac{\alpha^{2}}{2}\right), & \alpha \geq 0, \\ 0, & \alpha<0 .\end{cases}
$$

The transmitted signal is corrupted with a zero-mean additive white Gaussian noise (AWGN) $w(t)$ with two-sided power spectral density (PSD) $N_{0} / 2$ and a NBI modeled as

$$
I(t)=\sqrt{2 J} \cos \left(\omega_{c} t+\phi(t)\right)
$$

where $J$ is the power of the interference and $\phi(t)$ is the instantaneous phase deviation.

The received signal $r(t)$ at the input of the correlator bank in Figure 1 is expressed as

$$
r(t)=\alpha \sum_{i=1}^{K} s_{i}(t-\tau)+w(t)+I(t)
$$

At time $k T_{c}$ the samples $x^{I}\left(k T_{c}\right)$ and $x^{Q}\left(k T_{c}\right)$ in Figure 1 can be written as (for simplicity, $T_{c}$ is normalized to unity)

$$
\begin{aligned}
& x^{I}(k)=s^{I}(k)+n^{I}(k)+\zeta^{I}(k) \\
& x^{Q}(k)=s^{Q}(k)+n^{Q}(k)+\zeta^{Q}(k) \\
& s^{I}(k)=\alpha \sqrt{\frac{P}{4}} \sum_{i=1}^{K} b_{i, k}^{I} c_{i, k}^{I} \\
& s^{Q}(k)=\alpha \sqrt{\frac{P}{4}} \sum_{i=1}^{K} b_{i, k}^{Q} c_{i, k}^{Q}
\end{aligned}
$$

where

$b_{i, k}^{I} \in\{+1,-1\}$ and $b_{i, k}^{Q} \in\{+1,-1\}$ are the values of data signals at the $k$-th sampling instant of the $i$-th user for the in-phase and quadrature-phase components.

$c_{i, k}^{I} \in\{+1,-1\}$ and $c_{i, k}^{Q} \in\{+1,-1\}$ are the values of the spectrum spreading signals at the $k$-th sampling instant for the $i$-th user for the in-phase and quadrature-phase components, respectively.

$n^{I}(k)$ and $n^{Q}(k)$ are independent and uncorrelated random processes with zero mean and variance $\sigma^{2}=\frac{N_{0}}{4 T_{c}}$.

Assuming $\phi(t)$ to be varying slowly such that it is constant over one chip interval, then NBI components $\zeta^{I}(k)$ and $\zeta^{Q}(k)$ in (11) and (12) are expressed as

$$
\begin{aligned}
& \zeta^{I}(k)=\sqrt{\frac{J}{2}} \cos \phi(k) \\
& \zeta^{Q}(k)=\sqrt{\frac{J}{2}} \sin \phi(k)
\end{aligned}
$$

It can easily be shown that $s^{I}(k)$ and $s^{Q}(k)$ are zero-mean uncorrelated random processes each with variance $\sigma_{s}^{2}=E\left[\alpha^{2}\right] \frac{P}{4} K$.

\section{Complex Coefficient Adaptive IIR Notch Filter}

Using complex notation, a complex input signal to a complex coefficient adaptive IIR notch filter in Figure 1 is of the form

$$
x(k)=s(k)+\zeta(k)+n(k),
$$

where

$$
\begin{aligned}
& s(k)=s^{I}(k)+j s^{Q}(k) \\
& \zeta(k)=\sqrt{\frac{J}{2}} \exp (j \phi(k)) \\
& \phi(k)=\phi(k-1)+\omega(k),
\end{aligned}
$$




$$
n(k)=n^{I}(k)+j n^{Q}(k) .
$$

$\omega(k)$ is the instantaneous frequency that follows a random walk model and is expressed as

$$
\omega(k)=\omega(k-1)+\beta \nu(k), \omega(0)=\omega_{0}
$$

where $v(k)$ is a zero-mean white noise with variance $\sigma_{v}^{2}, \beta$ is the scaling factor for the frequency drift and is assumed to be small, i.e., $\beta \ll 1$ and $\omega_{0}$ is the initial frequency.

Transfer function of first-order complex coefficient IIR notch filter for suppression of the NBI in (17) is given by [11].

$$
H(z)=\frac{1+\alpha_{0}}{2} \frac{1-e^{j \alpha_{1}(k)} z^{-1}}{1-\alpha_{0} e^{j \alpha_{1}(k)} z^{-1}}
$$

where $\alpha_{0}$ is the notch bandwidth coefficient and $\alpha_{1}$ is the notch frequency coefficient. Adaptation algorithm used here to estimate the instantaneous frequency of the NBI is expressed as [11].

$$
\alpha_{1}(k+1)=\alpha_{1}(k)+\mu \operatorname{Re}\left[e(k) \varphi^{*}(k)\right] \text {. }
$$

$\operatorname{Re}[$.$] denotes real number and * denotes complex conju-$ gate. Here $\mu$ is the step size constant, $e(k)$ is the complex notch filter output and $\varphi(k)$ is the gradient signal. Referring to (23), the instantaneous frequency can be estimated by $\hat{\omega}(k)$ where

$$
\hat{\omega}(k)=\alpha_{1}(k) \text {. }
$$

Transfer function from the input $x(k)$ to the gradient signal $\varphi(k)$ in (24) is given by [11].

$$
G(z)=\frac{1+\alpha_{0}}{2} \frac{j e^{j \alpha_{1}(k)} z^{-1}}{1-\alpha_{0} e^{j \alpha_{1}(k)} z^{-1}} .
$$

\section{Frequency Tracking Error Analysis}

In this section, frequency tracking error of the algorithm in (24) is analyzed. We define steady-state tracking error of instantaneous frequency $\delta(k)$ as [14], [15].

$$
\delta(k)=\hat{\omega}(k)-\omega(k) .
$$

Referring to the coefficient adaptation algorithm in (24), it follows from (27) that [15]:

$$
\delta(k+1)=\delta(k)-\beta v(k)+\mu \operatorname{Re}\left[e(k) \varphi^{*}(k)\right],
$$

A first-order difference equation with respect to $\delta(k)$ is obtained by using approximations for steady-state signals $e(k)$ and $\varphi(k)$ derived in [14], and is expressed by

$$
\delta(k+1)=\left(1-\frac{J}{2} \frac{\mu}{4 \gamma^{2}}\right) \delta(k)+\mu \xi(k), \gamma=\frac{1-\alpha_{0}}{1+\alpha_{0}}
$$

where the input to the difference equation $\xi(k)$ is given by

$$
\begin{aligned}
\xi(k)= & \sqrt{\frac{J}{2}} \frac{1}{4 \gamma}\left\{n_{e}(k) e^{-j\left(\phi(k)+\frac{\pi}{2}\right)}+n_{e}^{*}(k) e^{j\left(\phi(k)+\frac{\pi}{2}\right)}\right\} \\
& +\frac{1}{2}\left\{n_{e}(k) n_{\varphi}^{*}(k)+n_{e}^{*}(k) n_{\varphi}(k)\right\}-\frac{\beta \nu(k)}{\mu}
\end{aligned}
$$

Here $n_{e}(k)$ and $n_{\varphi}(k)$ are the output of $H(z)$ and $G(z)$, respectively, due to $s(k)+n(k)$. By referring to (29), a first-order real coefficient transfer function $F(z)$ between the input $\xi(k)$ and the output $\delta(k)$ is given by

$$
F(z)=\frac{\mu z^{-1}}{1-\left(1-\frac{J}{2} \frac{\mu}{4 \gamma^{2}}\right) z^{-1}} .
$$

\subsection{Square of Tracking Error Due to Frequency Drift}

By referring to (29), (30) and (31), square of tracking error due to the frequency drift is found to be given by

$$
\sigma_{1}^{2}=\sigma_{v}^{2}\left(\frac{\beta}{\mu}\right)^{2} \frac{1}{2 \pi j} \oint F(z) F\left(z^{-1}\right) z^{-1} d z=\frac{2}{J} \frac{2 \sigma_{v}^{2} \beta^{2} \gamma^{2}}{\mu}
$$

(32) is obtained by assuming that $\frac{J}{2} \mu \ll 4 \gamma^{2}$ which means the step-size parameter is sufficiently small.

\subsection{Frequency Tracking Mean Square Error}

Frequency tracking mean square error (MSE) is the sum of $\sigma_{1}^{2}$ in (32) and frequency error variance $\sigma_{2}^{2}$ due to $n_{e}(k)$ and $n_{\varphi}(k) . \sigma_{2}^{2}$ is obtained by $[12,14]$.

$$
\begin{gathered}
\sigma_{2}^{2} \approx \frac{\frac{J}{2}\left(E\left[\alpha^{2}\right] \frac{P}{2} K+\frac{N_{0}}{2 T_{c}}\right) \mu^{2}\left(1+\alpha_{0}\right)^{3}}{16\left(1-\alpha_{0}\right)^{2}\left(1-\alpha_{0} \varepsilon\right)(1+\varepsilon)}, \\
\varepsilon=1-\frac{J}{2} \frac{\mu}{4 \gamma^{2}} \approx 1,
\end{gathered}
$$

The following observations are made from (33) and (34):

1) Frequency tracking error variance is directly proportional to the NBI power $J$.

2) For small AWGN PSD $N_{0}$ frequency tracking error variance is directly proportional to the signal transmit power $P$ and number of active users $K$.

3) Frequency tracking error variance can be reduced by expanding the notch bandwidth, i.e., reducing the 
notch bandwidth coefficient $\alpha_{0}$.

4) Frequency tracking error variance varies proportionally with the square of step-size constant.

From (32), (33) and (34), the closed-form expression for the frequency tracking MSE can be expressed as

$$
M S E=\frac{2}{J} \frac{2 \sigma_{v}^{2} \beta^{2} \gamma^{2}}{\mu}+\frac{\frac{J}{2}\left(E\left[\alpha^{2}\right] \frac{P}{2} K+\frac{N_{0}}{2 T_{c}}\right) \mu^{2}}{32 \gamma^{3}} .
$$

\subsection{Optimum Step Size and Notch Bandwidth Coefficients}

From (35), the optimum step-size $\mu_{\text {opt }}$ that corresponds to minimum frequency tracking $\mathrm{MSE}$ is derived by equating the first derivative of MSE in (35) with respect to $\mu$ to zero. This yield:

$$
\mu_{o p t}=2\left(\frac{4 \sigma_{v}^{2} \beta^{2} \gamma^{5}}{\left(\frac{J}{2}\right)^{2}\left(E\left[\alpha^{2}\right] \frac{P}{2} K+\frac{N_{0}}{2 T_{c}}\right)}\right)^{\frac{1}{3}} .
$$

Similarly, equating to zero the first derivative of MSE in (35) with respect to $\gamma$ we obtain:

$$
\alpha_{0 o p t}=\frac{1-\gamma_{o p t}}{1+\gamma_{o p t}} \text {. }
$$

where:

$$
\gamma_{o p t}=\frac{1}{2}\left(\frac{3\left(\frac{J}{2}\right)^{2}\left(E\left[\alpha^{2}\right] \frac{P}{2} K+\frac{N_{0}}{2 T_{c}}\right) \mu^{3}}{4 \sigma_{v}^{2} \beta^{2}}\right)^{\frac{1}{5}}
$$

\section{Simulation and Discussions}

In this section, computer simulation results are compared with analytical values obtained in (35), (36), (37) and (38) to substantiate the accuracy of the proposed analytical method. All simulated results were obtained by averaging over 50 independent computer runs for $k=2500$ to 14000 with $J=2.0$, and $P=0.1$, i.e., interference-to-signal power ratio (ISR) of $13.0 \mathrm{~dB}$. The values for $\beta, \sigma_{\mathrm{v}}^{2}$, and $\omega_{0}$ were set to $10^{-3}, 0.2$, and $0.2 \pi$, respectively, and $L=255$. Here the AWGN PSD $N_{0}$ is ignored as it is assumed to be much smaller than the NBI power $J$.

In Figure 2, simulated results for the frequency track- ing MSE are plotted and compared with theoretical values obtained from (29) with the DS-CDMA system number of active users $K$ as a parameter. Here the step size constant $\mu$ was set to $10^{-4}$. For small values of $\alpha_{0}$, the figure shows a decrease in the frequency tracking MSE which increases after $\alpha_{0}$ exceeds the optimal notch bandwidth coefficient $\alpha_{\text {opt }}$ whose value depends on $K$. Close agreement between analytical and simulation results is clearly demonstrated by the figure.

Figure 3 shows plots of theoretical values of the frequency tracking MSE obtained from (35) and simulated results with the number of active DS CDMA users K as a parameter for $\alpha_{0}=0.90$. It is observed from the figure that the frequency tracking MSE decreases for values of $\mu$ less than the optimal step size $\mu_{\text {opt }}$ which depends on $K$. For values of $\mu$ above $\mu_{\text {opt }}$, the frequency tracking MSE increases steadily. Similarly, close agree-

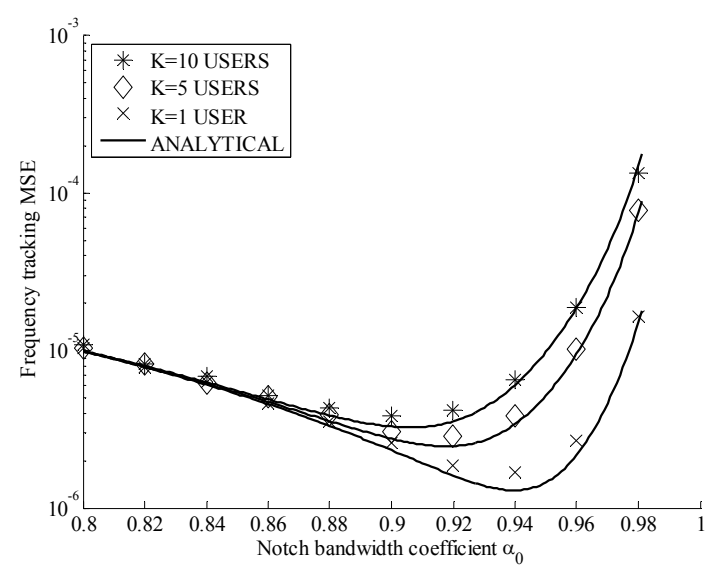

Figure 2. Frequency tracking MSE with number of DS-CDMA users $K$ as a parameter.

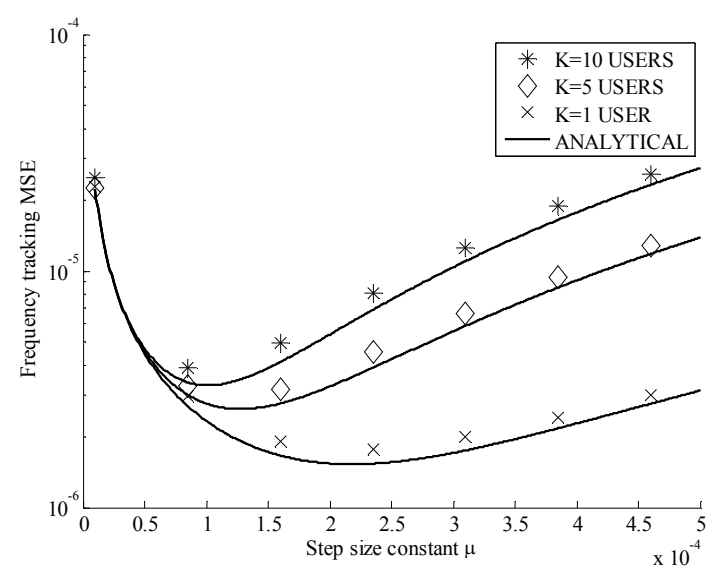

Figure 3. Frequency tracking MSE with number of DS-CDMA active users $K$ as a parameter. 
ment between analytical values and simulation results is clearly shown by the figure.

Figure 4 shows simulated results and theoretical values for the optimum notch bandwidth coefficient $\alpha_{0_{\text {opt }}}$ plotted against the number of DS-CDMA active users $K$ for $\mu=10^{-4}$. Similarly, the figure shows a decrease in $\alpha_{0_{\text {opt }}}$ with the increase in $K$ as predicted by (37) and (38). There is a close agreement between simulated results and theoretical values as validated by the figure.

Simulated results and theoretical values obtained from (36) for the optimum step-size constant $\mu_{\mathrm{opt}}$ versus number of active DS-CDMA users $K$ are plotted in Figure 5 with $\alpha_{0}=0.9$. The figure shows a decrease in $\mu_{\text {opt }}$ with the increase in $K$ as anticipated by (30). Close agreement between simulated results and theoretical values is clearly demonstrated by the figure.

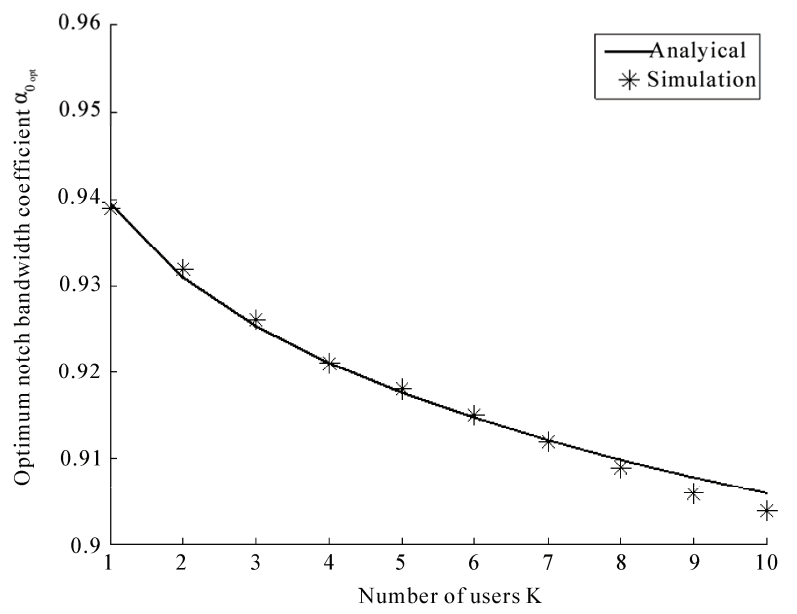

Figure 4. Optimum step-size constant versus number of active users $K$.

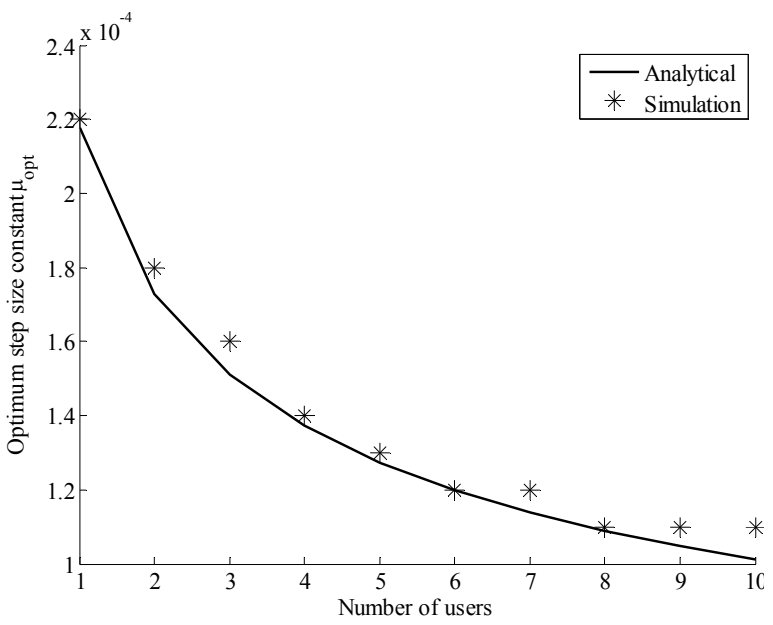

Figure 5. Optimum notch bandwidth coefficient versus the number of DS-CDMA active users $K$.

\section{Conclusions}

Frequency tracking characteristics of the complex-coefficient adaptive IIR notch filter for suppression of NBI with randomly-varying frequency in a DS-CDMA communication system over a Rayleigh fading channel were investigated in this paper. Derived closed-form expressions for frequency tracking MSE and optimum step size and notch bandwidth coefficient have revealed a need for proper setting of adaptation algorithm and IIR notch filter parameters to minimize frequency tracking MSE. Moreover, computer simulation results have demonstrated the accuracy of the analytical approach. In the future, probability of bit error of the DS-CDMA system with NBI suppression complex adaptive IIR notch filter will be investigated.

\section{References}

[1] W. C. Y. Lee, "Overview of cellular CDMA," IEEE Transactions on Vehicular Technology, Vol. 40, pp. 291301, May 1991

[2] R. L. Pickholtz, D. L. Schilling, and L. B. Milstein, "Theory of spread spectrum communications: A tutorial," IEEE Transactions on Communications, Vol. COMM-30, pp. 855-884, May 1982.

[3] L. B. Milstein, et al., "On the feasibility of a CDMA overlay for personal communications networks," IEEE Journal on Selected Areas in Communications, Vol. 10, pp. 655-668, May 1992.

[4] H. V. Poor and L. A. Rusch, "Narrowband interference suppression in spread spectrum CDMA," IEEE Communications Magazine, Vol. 1, pp. 14-27, August 1994.

[5] J. Wang and L. N. Miltein, "Adaptive LMS filters for cellular CDMA overlay situations," IEEE Journal on Selected Areas in Communications, Vol. 14, pp. 1548-1559, October 1996

[6] M. Lops, G. Ricci, and A. M. Tulino, "Narrowband interference suppression in multiuser CDMA systems," IEEE Transactions on Communications, Vol. 46, pp 1163-1175, September 1998.

[7] S. Buzzi, M. Lops, and A. M. Tulino, "Time-varying narrow-band interference rejection in asynchronous multiuser DS/CDMA systems over frequency-selective fading channels," IEEE Transactions on Communications, Vol. 47, pp. 1523-1535, October 1999.

[8] W. S. Hou, L. M. Chen and B. S. Chen, "Adaptive narrowband interference rejection in DS-CDMA systems: A scheme of parallel interference cancellers," IEEE Journal on Selected Areas in Communications, Vol. 19, pp. 1103-1114, June 2001

[9] V. Krishnamurthy, G. Yin, and S. Singh, "Adaptive step-size algorithms for blind interference suppression in DS/CDMA systems," IEEE Transactions on Signal Processing, Vol. 49, pp. 190-201, January 2001. 
[10] S. C. Pei and C. C. Tseng, "Complex adaptive IIR notch filter algorithm and its applications," IEEE Transactions on Circuits and Systems, Vol. CAS-41, No. 2, pp. 158163, February 1994.

[11] S. Nishimura and H. Y. Jiang, "Simplified realization of cascaded adaptive notch filters with complex coefficients," Proceedings of International Symposium on Circuits and Systems, Vol. 5, pp. 269-272, 1998.

[12] H. Y. Jiang, S. Nishimura, and T. Hinamoto, "Convergence analysis of complex adaptive IIR notch filters for the detection of single sinusoid," IEICE Transactions Fundamentals, Vol. E82-A, No. 6, pp. 912-915, June 1999.
[13] H. Y. Jiang, S. Nishimura, and T. Hinamoto, "Steadystate analysis of complex adaptive IIR notch filter and its application to QPSK communication systems," IEICE Transactions Fundamentals, Vol. E85-A, No. 5, pp. 1088-1085, May 2002.

[14] A. Mvuma, S. Nishimura, and T. Hinamoto, "Tracking analysis of complex adaptive IIR notch filter for a linear chirp signal," IEICE Transactions Fundamentals, Vol. E92-A, pp. 1526-1529, June 2009.

[15] A. Mvuma, S. Nishimura, and T. Hinamoto, "Complex coefficient adaptive IIR notch filter tracking characteristics," Proceedings of Midwest Symposium on Circuits and Systems, pp. 640-643, August 2009. 